\title{
Representaciones sociales sobre la escritura de la tesis en dos carreras del área de humanidades: Periodismo y Trabajo Social
}

\author{
Representações sociais da escrita de teses \\ em dois programas de Ciências Humanas: \\ Jornalismo e Serviço Social
}

Social Representations about Thesis Writing in Two Majors in Humanities: Journalism and Social Work

Monica Tapia Ladino*

Universidad Católica de La Santísima Concepción

Concepción / Chile

Juanita Marinkovich**

Pontificia Universidad Católica de Valparaíso

Valparaíso / Chile

RESUMEN: Las representaciones que sobre las tareas de escritura tienen tanto estudiantes como profesores son consecuencia de sus propias experiencias y expectativas sociales. El objetivo de este artículo es relevar cómo son las representaciones sociales de docentes que guían tesis y estudiantes que han elaborado tales escritos en dos carreras del área de humanidades de una universidad chilena. Se aplicó la técnica del grupo focal a ocho docentes universitarios de las humanidades y seis entrevistas individuales a estudiantes tesistas. Los datos se analizaron siguiendo los principios de la teoría empiricamente fundada. Los resultados revelan que la escritura es una tarea que define la formación y determina la identidad profesional. La tesis de grado se asimila a los rasgos de los textos profesionales de los campos para los cuales se forman los estudiantes.

PALABRAS CLAVE: Escritura académica; tesis; comunidades discursivas; representaciones sociales.

\footnotetext{
*mtapia@ucsc.cl

**jmarinko@ucv.cl
} 
RESUMO: As representaçōes que tanto estudantes quanto professores têm sobre as tarefas de escritura são consequência das suas próprias experiências e expectativas sociais. O objetivo deste artigo é revelar como são as representaçóes sociais dos professores que orientam teses e dos alunos que desenvolvem tais escritos em dois programas da área de humanidades numa universidade chilena. Aplicou-se a técnica do grupo focal a oito professores universitários das humanidades e seis entrevistas individuais com alunos em tese. Os dados foram analisados de acordo com os princípios da teoria fundada. Os resultados revelam que escrever é uma tarefa que define a formação e determina a identidade profissional. A tese é equiparada à categoria das características dos textos profissionais dos campos para os quais os alunos são formados.

PALAVRAS-CHAVE: Escrita acadêmica; teses; comunidades discursivas; representaçôes sociais.

ABSTRACT: The representations that teachers and students have about writing tasks are a consequence of their own experiences and social expectations. The aim of this paper is to notice how the social representations of the teachers who guide research thesis and students who have elaborated these pieces of writing in two Chilean university programs in the area of social sciences are. Focus group technique was applied to 8 university teachers of the social science area and 6 individual interviews to thesis students. The data was analysed following the principles of Grounded Theory. The results show that writing is a task which defines academic development and determines professional identity. The thesis to obtain a degree is similar to the traits of a professional text of the areas to which students are formed.

KEYWORDS: Academic writing; thesis; discourse communities; social representations.

\section{Antecedentes}

Nos interesa estudiar las representaciones sociales (RS), porque la actividad de escribir forma parte de una experiencia central de las comunidades discursivas académicas. El supuesto que subyace a este interés tiene que ver con la idea de que la escritura no es sólo una actividad cognitiva, sino también un fenómeno social sobre el cual los integrantes de una comunidad desarrollan ideas y pensamientos compartidos. Un modo de comprender la naturaleza del fenómeno de la escritura es acceder al mundo representacional de las ideas y percepciones que ella provoca en quienes emprenden la tarea de producir un texto de envergadura como es la tesis de licenciatura.

Como la universidad busca formar personas para que se desenvuelvan preferentemente en el mundo laboral, este representa el espacio que articula la formación escolar y la formación profesional. El objetivo de los estudios universitarios consiste, por un lado, en favorecer la inmersión en el espacio 
académico y, por otro, preparar a los estudiantes para el adecuado desempeño en el mundo laboral. Desde esta perspectiva, los estudiantes universitarios requieren aprender tanto las materias relacionadas con los campos disciplinares específicos de su formación como aquellas que son necesarias para desenvolverse adecuadamente en el mundo laboral. Por lo tanto, a los estudiantes les corresponde leer y escribir para comprender las disciplinas que son la base de su formación para luego, en las carreras que otorgan título y grado, producir textos típicos que les serán exigidos en el ámbito profesional (CARLINO, 2005; PARODI, 2008; TAPIA-LADINO; SILVA, 2010).

Las bases teóricas de esta investigación provienen de los aportes de la alfabetización académica, literacidad y letrismo, de las representaciones sociales y de los géneros discursivos académicos y profesionales.

\section{Alfabetización académica, literacidad y letrismo}

Uno de los conceptos que ha servido para explicar las disonancias entre la formación escolar y la formación universitaria, ha sido el de alfabetización académica. Este concepto refiere al "conjunto de nociones y estrategias necesarias para participar en la cultura discursiva de las disciplinas así como en las actividades de producción y análisis de textos requeridos para aprender en la universidad" (CARLINO, 2005, p. 13-14). Desde esta perspectiva, es comprensible la disonancia que se produce entre los modos de leer y escribir en el mundo escolar con las maneras exigidas en la universidad. En la universidad, los estudiantes necesitan abordar textos que presentan rasgos de polifonía y autoría diferentes a los planteados en el sistema escolar donde normalmente no expresan polémica ni autorías. Tal discrepancia puede explicar el hecho de que los estudiantes universitarios presenten serios problemas para comprender y producir textos académicos. Dichas dificultades han sido largamente diagnosticadas por diferentes autores. ${ }^{1}$

En Chile, encontramos también algunos trabajos, como es el caso de Mónica Tapia, Gina Burdiles y Beatriz Arancibia (2003), que plantea que los estudiantes universitarios evidencian en sus informes el uso de la reproducción textual de fragmentos de las fuentes, en desmedro del procesamiento de la

${ }^{1}$ Lucy McCormick (1997); Donald M. Murray (1997); Guglielmo Cavallo y Roger Chartier (1998); Virginia Narvaja de Arnoux et al. (1998); Fernando Romero (1998; 2000); Adriana Bono y Sonia de la Barrera (1998); Natalia B. Cano (1999); Narvaja de Arnoux, Mariana Di Stefano y Cecilia Pereira (2002), entre otros. 
información para crear textos autónomos, intencionados, que (re)construyan conocimiento. Por su parte, Carolina Espejo Repetto (2006), desde un punto de vista más descriptivo analiza algunas de las estrategias retóricas (moves, en términos de John Swales, 1990) empleadas por alumnos universitarios de tres diferentes áreas, al redactar la sección "Conclusión" de los artículos de investigación. Sus resultados revelan que los distintos movimientos retóricos relevantes para una investigación evidenciaron un conocimiento disímil de las convenciones del género informe. Las razones parecen relacionarse con la dificultad para expresarse de manera más formal así como el diferente nivel de formación de los estudiantes.

De igual relevancia son los aportes de Ana María Harvey (2005), Harvey y Daniel Muñoz (2006), Paulina Núñez (2004) y Núñez y Espejo (2005), quienes indagan en las características y los estilos de las comunidades discursivas en las cuales se producen los textos académicos. Los hallazgos de las autoras revelan que los estudiantes tienen conciencia del valor formativo de los informes académicos. Asimismo, textos académicos como los informes cumplen una función evaluativa preponderante en la formación superior. Los hallazgos evidencian que las actividades relacionadas con la lectura y la escritura no son sólo cognitivas, sino parte de prácticas sociales de grupos determinados de personas.

Desde hace algunos años, se ha propuesto el concepto de literacidad para explicar la dimensión sociocultural del fenómeno de la lectura y la escritura. Para Virginia Zavala la literacidad "implica una manera de usar la lectura y la escritura en el marco de un propósito social específico" (ZAVALA, 2009, p. 23). De este modo, se trata de una idea que supera el conocimiento y manejo del código escrito para atender los objetivos sociales que mueven a los hablantes a usar la lectura y la escritura para desarrollar alguna tarea. Para los investigadores (CASSANY, 2005; GONZÁLEZ et al., 2009) la literacidad tiene un sentido más amplio que el de alfabetismo, pues incluye los aspectos del código de la lengua, los géneros discursivos, los roles del autor y del lector, las formas de concebir la realidad, la identidad, los valores y las representaciones culturales que emanan del uso de la lengua escrita. Por lo tanto, si adoptamos esta perspectiva de análisis no basta con estudiar la literacidad sólo desde una perspectiva lingüística, sino que además es necesario atender a las manifestaciones que produce la interacción interpersonal a partir de lo que las personas hacen con los textos.

En la misma línea de la escritura como una manifestación sociocultural, Vargas Gerardo Del Rosal propone el concepto letrismo para referirse "al 
modelo de funcionamiento de los sistemas simbólicos, especialmente de la lengua escrita, en la conformación de comunidades profesionales de la lengua escrita, en la conformación de comunidades profesionales y/o científicas" (DEL ROSAL, 2009, p. 12). El letrismo, según el autor, explica los procesos de configuración y los modos de comprender subjetivos que son regulados por modelos validados por una comunidad. Zavala, por su parte, agrega una expresión asociada denominada prácticas letradas con la que quiere expresar la relación entre las actividades y los textos, es decir, "las formas culturales generalizadas de uso de la lengua escrita [...] maneras de leer y escribir" (ZAVALA, 2009, p. 26). De esta manera, leer y escribir son modos particulares de construir la realidad que sólo tienen sentido dentro de una comunidad particular. Las prácticas que cada comunidad realiza van configurando su identidad de tal modo que escribir, para el caso que nos interesa, es una actividad que se convierte en el vehículo que permite identificarse como participante de una comunidad. Para Elvira Narvaja de Arnoux (2009) el concepto de letrismo viene a superar el de alfabetización académica dado que esta idea pone mayor énfasis en la naturaleza sociocultural como fenómeno interrelacionado y no como sistemas de individuos que actúan aisladamente.

Estamos de acuerdo con Daniel Cassany (2005; 2009), Zavala (2009) y Narvaja de Arnoux (2009) con la idea de que leer y escribir son prácticas sociales producto de un propósito social. En este marco, la escritura se entiende como una actividad más compleja que el mero dominio del código como una actividad motora. Los autores están de acuerdo en que escribir en cualquier esfera de la vida es una acción en la que se reproducen sociales roles vinculados a instituciones. En este marco, leer y escribir adquieren valores que se rigen por relaciones de poder. Cuando escribimos asumimos un rol como autor y configuramos un lector que es parte de una comunidad. En la universidad precisamente la actividad de escribir una tesis de pregrado representa el paso necesario para la obtención de un título y/o un grado. Para llevar a cabo esta tarea, los escritores o autores de las investigaciones necesariamente requieren adoptar las convenciones escriturales de su comunidad.

Una tesis es un texto que presenta la investigación y los resultados de un autor apoyado por un académico guía para obtener un grado o un título profesional. La palabra tesis proviene del griego èÝóéo, que significa la 'posición', y se refiere a un asunto intelectual. La raíz etimológica latina thesis, por su parte, significa 'tema', por lo que entendemos que se trata de una postura intelectual acerca de un tópico particular. 
La escritura académica de la cual forma parte la escritura tesis es una actividad de inclusión a las prácticas disciplinares y profesionales específicas. Dichos textos forman parte de los géneros especializados dado que se trata de escritos no cotidianos que se elaboran dentro de dominios conceptuales particulares que revelan una función básicamente referencial y cuyo contexto de circulación es una comunidad universitaria específica (PADRÓN, 1996; GUNNARSSON, 1997; TOLCHINSKY, 2000; ALDELSTEIN, 2000; PARODI, 2008; 2010). Dentro de los géneros especializados, reconocemos géneros académicos como aquellos que se elaboran preferentemente dentro del ámbito universitario. Como parte de su proceso de formación, los estudiantes universitarios requieren ingresar a la comunidad discursiva de su especialidad con el fin de mantener y extender el conocimiento de su área y así pasar a ser miembros del grupo. Para diversos autores (PARODI, 2008; VENEGAS, 2007), el discurso académico es epistémico y constitutivo del conocimiento del grupo, cuyos textos se organizan a través de un continuum en el que se entrelazan desde los textos escolares hasta profesionales. Por tanto, los estudiantes universitarios deben ser capaces de producir textos académicos, es decir, escritos con función epistémica sobre el conocimiento de su área, para generar la identidad tanto de la formación disciplinar como de la formación profesional.

Una manera de abordar una arista de los textos académicos, es acceder a las ideas que una comunidad discursiva se representa para comprender y concebir la actividad de escribir (MARINKOVICH; VELÁSQUEZ, 2010; TAPIA LADINO; MARINKOVICH, 2011). Como señala Jay L. Lemke (2001), lo importante al hacer ciencia son las tradiciones culturales socialmente aprendidas, los tipos de discurso y las representaciones acerca de cómo se usan. Tal conocimiento sería más útil que identificar sólo los mecanismos mentales que se activan al hacerlo.

\section{Representaciones sociales}

Para Émile Durkheim (1898) las representaciones colectivas son formas de conocimiento o ideación construidas socialmente que no pueden explicarse como fenómenos aislados. Para Serge Moscovici (1976) el concepto de representación social difiere del de representación colectiva, dado que el primero tiene un carácter más dinámico. Según el autor, las RS no son sólo productos mentales, sino que son construcciones simbólicas que se crean y recrean en el curso de las interacciones sociales. Moscovici define las RS como un 
conjunto de conceptos, declaraciones y explicaciones originadas en la vida cotidiana, en el curso de las comunicaciones interindividuales. Equivalen, en nuestra sociedad, a los mitos y sistemas de creencias de las sociedades tradicionales; puede, incluso, afirmarse que son la versión contemporánea del sentido común. (MOSCOVICI, 1981, p. 181).

Las representaciones sociales son actividades mentales que favorecen la relación con situaciones, acontecimientos y las personas (JODELET, 1989). Según Moscovici (1976), las RS son sistemas de ideas que se originan en la vida diaria. Ellas se configuran en tres dimensiones: información, actitudes y campo de representación. La información se relaciona con la organización de las ideas en un grupo sobre un objeto social, un contenido real y acotado de las proposiciones sobre las representaciones. Las actitudes se relacionan con la focalización que se realiza en torno a un objeto o suceso.

Las representaciones sociales son comprendidas como formas de conocimiento que le permiten al sujeto y a su grupo volver parte de su mundo interior a un objeto nuevo y extraño que genera desequilibrio. Las RS se caracterizan porque son generadas por los sujetos sociales. Se conciben como una producción y elaboración de carácter social no impuesta externamente, pues conforman un conocimiento espontáneo, práctico, un producto y proceso de la actividad.

Como nos interesa dar cuenta de las ideas que sobre un fenómeno tienen un grupo de personas que comparten un ámbito de formación, nos referimos a representaciones colectivas que no son el resultado de experiencias individuales, sino el efecto de la cooperación e interrelación que se produce en los sujetos participantes de una experiencia, lo que provoca alteraciones recíprocas (ALVAREZ, 1996). El interés por conocer esta área de la realidad se basa en el supuesto de que las representaciones sociales que los hablantes conciben a propósito de la experiencia sobre la escritura académica determina el modo como enfrentan dicha tarea y tendría consecuencias en la construcción lingüísticodiscursiva de sus textos. Por lo tanto, en este trabajo se intenta describir las concepciones acerca de la escritura académica y sobre el género discursivo tesis que vivencian una comunidad de especialistas del área de las humanidades.

\section{Escritura académica}

Como sabemos, la escritura requerida en la universidad es una práctica académica que varía según las culturas institucionales en las que tiene lugar, de acuerdo a concepciones compartidas por sus miembros, generalmente en 
forma tácita. Cada disciplina académica posee su propio método para organizar el conocimiento y dispone de formas específicas para escribirlo. Este supuesto es abordado por el movimiento denominado "escritura en las disciplinas", que se preocupa por comprender qué ocurre con la escritura en diferentes disciplinas y el movimiento de reforma curricular para ofrecer una instrucción relacionada disciplinariamente en un programa específico diseñado para tal propósito (BAZERMAN, 2005). Este movimiento forma parte de uno más amplio denominado escritura a través de currículum, cuyo objetivo es relacionar la actividad de escribir con el aprendizaje. Estos enfoques buscan que los estudiantes adopten progresivamente el discurso académico de sus disciplinas a través del texto escrito (MARINKOVICH; MORÁN, 1998).

En una línea similar, Enrique Alcaraz Varó et al. proponen que las convenciones discursivas propias de áreas disciplinares se pueden corresponder con la idea de lenguas. De ese modo, sostiene que las lenguas de las "comunidades epistemológicas son lenguas profesionales, pero también son académicas porque antes de haber sido utilizadas en cada ambiente profesional, fueron enseñadas y aprendidas en la universidad" (ALCARAZ VARÓ et al., 2007, p. 7). Para el autor, en la universidad es posible identificar los movimientos epistemológicos de las disciplinas en los que se advierte un movimiento recíproco en el que los textos académicos influyen a los textos profesionales y viceversa. Se trataría de un movimiento de flujo y reflujo. Estas ideas expresan la relación entre la formación y las exigencias del mundo laboral.

Entre los textos académicos que se producen en el ámbito universitario podemos distinguir los escritos disciplinares y los profesionales. Los primeros son necesarios para expresar el dominio de las convenciones lingüístico-discursivas con las cuales se vehicula el saber propio de una disciplina de estudio. Siguiendo a Giovanni Parodi (2008), se trata de textos en los que se expresa el saber de un conjunto deáreas. Por su parte, los segundos son aquellos que se elaboran en la rutina laboral para la cual se prepara el estudiante en la universidad. Dichos escritos permiten la comunicación eficiente entre los profesionales y el funcionamiento de un área de desarrollo. De este modo y siguiendo al mismo autor, el discurso académico está vinculado a saber disciplinas o materias y los discursos profesionales están orientados al haceren el ámbito laboral. Desde esta perspectiva, la presente investigación aborda el estudio de la tesis o seminario final de grado, pues es el escrito más extenso y complejo que los estudiantes producen para acreditar el saber de las disciplinas de formación. El objetivo del estudio es identificar las RS de estudiantes y profesores universitarios del área de las humanidades (Periodismo y Trabajo Social) sobre el proceso de la escritura 
universitaria y la escritura de la tesis de pregrado siguiendo los principios de la metodología de la teoría fundamentada (TF).

\section{Metodología}

El enfoque metodológico seleccionado corresponde al que propone la TF (GLASER; STRAUSS, 1967; STRAUSS; CORBIN, 2002). Se trata de una investigación de carácter cualitativo interpretativa que tiene como objetivo construir un marco teórico que explique algún fenómeno social, psicológico y educativo. El acceso a los procesos subjetivos de docentes y estudiantes por medio de una metodología cualitativa permitió construir conocimientos teóricos sobre las RS asociadas al proceso de escribir la tesis de pregrado de forma estructurada y flexible a partir de los datos obtenidos (RUIZ, 1996). En este caso, se estudian los discursos para explicar quién, qué, cuándo, dónde, por qué y con qué resultados ocurren los procesos.

\section{Los participantes}

El presente estudio se realizó con un grupo de docentes de planta y estudiantes tesistas de Periodismo y Trabajo Social de una universidad del Consejo de Rectores de Chile. Esta investigación representa una parte de una investigación mayor que busca averiguar lo que sucede con la escritura en las disciplinas en la etapa final de la formación académica de las licenciaturas en ciencias y humanidades en universidades de Chile. Para efectos de este artículo, presentamos una parte del trabajo relacionado con el abordaje al área de las humanidades.

Para ello, se seleccionaron doce casos o participantes que cumplían con los criterios de inclusión para el estudio: a) profesores que guían tesis de pregrado y b) estudiantes que escribieron una tesis de pregrado. Todos los académicos participantes son profesores permanentes y poseen estudios de posgrado diferentes (magíster o doctorado). Los estudiantes, por su parte, habían terminado de escribir sus tesis y se encontraban en proceso de obtención de sus títulos.

La recolección de los datos se realizó en dos etapas. En primer término, realizamos dos focus group (FG) - uno por carrera - a cuatro académicos de Periodismo: dos mujeres y dos varones, y tres profesores de Trabajo Social. Las conversaciones grupales se realizaron por un lapso de una hora. Antes de realizarlas, los docentes accedieron a participar mediante la firma de un 
consentimiento informado. Los temas abordados trataron sobre la función que asignan los docentes a los trabajos escritos, el modo como la enseñan, la función y características de la tesis, los tipos y rasgos de los escritos que solicitan antes de la tesis, la percepción que tienen sobre la calidad de la escritura de sus estudiantes, entre otros.

Por su parte, se realizaron seis entrevistas individuales a estudiantes - tres estudiantes varones de Periodismo y dos mujeres y un hombre de Trabajo Social. Las entrevistas duraron alrededor de 30 minutos y abordaron temas como tipos de escritos elaborados durante la carrera, proceso de aprendizaje de dichos textos, experiencia con la escritura de la tesis, dificultades y facilidades de la tarea, entre otros. Todas las conversaciones fueron registradas en grabadoras digitales, material que posteriormente fue transcrito a ortografía corriente.

Tanto los FG como las entrevistas personales a los estudiantes se realizaron con la técnica de la entrevista semiestructurada, lo que permitió que los participantes abordaran temas no contemplados, pero relacionados con el protocolo de preguntas.

\section{Análisis de los datos}

El análisis se realizó siguiendo los pasos propuestos por la TF, lo que permitió construir modelos conceptuales con respecto al fenómeno en estudio. La contribución de la TF al análisis cualitativo

dirige la atención hacia el proceso de conceptualización basado en la emergencia de patrones sociales a partir de los datos de la investigación. Este proceso cumple dos requisitos básicos desde la aproximación de la Teoría Fundamentada. Primero, los conceptos son abstractos en relación con el tiempo, los lugares y las personas, y, en segundo lugar, los conceptos son perdurables en su alcance teórico. (TRINIDAD; CARRERO; SORIANO, 2006, p. 10).

Para la realización de este estudio, se utilizó un método de análisis textual cualitativo con apoyo computarizado. La codificación inicial de esta etapa se realizó con el apoyo del software QSR NVivo, que facilitó la tarea de almacenamiento, sistematización y codificación de los textos. En general, la etapa de codificación abierta siguió el principio de comparación constante que pretende generar teoría mediante el análisis comparativo y sistemático de lo declarado por los sujetos (FLICK, 2004). La primera etapa fue la codificación 
abierta que consistió en el examen y fragmentación de la información recopilada para organizar los materiales en conceptos y categorías. Para ello, se siguió el principio de comparación constante que pretende generar teoría a partir del análisis comparativo y sistemático sobre lo declarado por los sujetos. Cada porción fue aislada por las investigadoras y asociadas a una categoría. En segundo término, se integraron las categorías y propiedades surgidas de la etapa anterior para formar el núcleo de la teoría emergente, actividad que correspondió a la codificación axial.

Para efectos de este artículo, trabajamos los datos considerando las carreras como criterios de comparación. La razón de esta decisión tiene que ver con el hecho de que un primer análisis mostró que los integrantes de cada carrera presentaban características comunes. A diferencia de trabajos previos (MARINKOVICH; VELÁSQUEZ, 2010; TAPIA LADINO; MARINKOVICH, 2011) donde comparamos entrevistas a estudiantes y académicos, en esta oportunidad hemos optado por comparar entre las entrevistas de profesores y estudiantes de las carreras de Periodismo y Trabajo Social, ya que los datos demostraron más rasgos comunes al interior de cada carrera que entre estudiantes y académicos.

Finalmente, los materiales fueron reducidos a una conceptualización teórica dado que la TF pone acento en la construcción y desarrollo de una teoría sustantiva, es decir, en un tipo de construcción teórica que surge a partir de los datos. Para concluir, se consideró la textualización de la teoría emergente mediante las anotaciones que se habían realizado durante las fases anteriores.

\section{Resultados}

Después de haber hecho las codificaciones pertinentes para llegar a la codificación axial, elaboramos un diagrama que muestra la relación que pudimos establecer entre las categorías. Para ello, trabajamos con las siguientes categorías: características de la tesis, dificultades en su proceso de escritura, apoyo al desarrollo de la escritura, función de los escritos en la formación, procesos de enseñanza-aprendizaje, tipos de textos exigidos en la formación, características de los escritos y escritura de los docentes.

En la TF se relacionan las categorías y propiedades mediante la alternancia de un pensamiento inductivo y deductivo. Se focaliza en la identificación de un fenómeno central y se distinguen las relaciones causales e intervinientes para explicar las relaciones dentro de un esquema organizativo de relaciones. El paradigma de codificación considera la identificación de los siguientes elementos: el fenómeno o idea central o incidente al que hacen 
referencia las acciones; el contexto, es decir, las condiciones bajo las cuales se da el fenómeno; las condiciones causales, constituidos por los sucesos que provocan la ocurrencia del fenómeno; las condiciones intervinientes que influyen sobre las estrategias de acción-interacción y que pueden facilitar o intervenir en ellos; las estrategias de acción que se utilizan para manejar o responder ante el fenómeno y las consecuencias que son los resultados de la acción e interacción del fenómeno.

A continuación, se despliega la codificación axial que contiene los elementos anteriormente señalados en torno al fenómeno o idea central relacionado con la comunidad de la carrera de Periodismo.

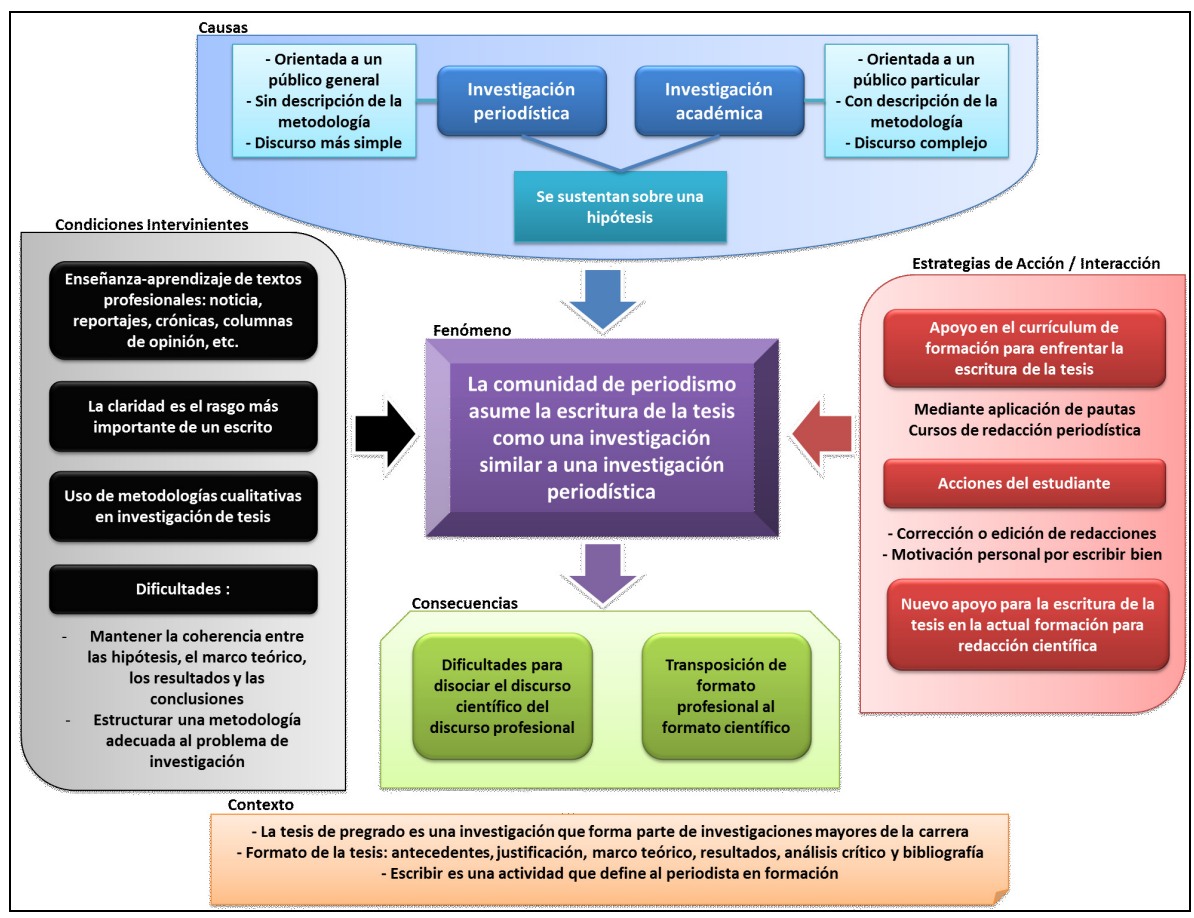

FIGURA 1 - Representaciones sociales de la comunidad de Periodismo Fuente: Elaboración propia.

Para la carrera de Periodismo, la tesis de final de carrera es una actividad concebida como un símil de la investigación periodística. El sustento de tal representación se basa en el hecho de que la investigación periodística también se realiza sobre la base de una hipótesis. Tales ideas se reflejan en los siguientes segmentos declarados por los académicos: 
[...] tu trabajo parte de una hipótesis, en un reportaje como en una tesis o un paper, tú partes de una hipótesis que tú al final la contrastes, eso es otra cosa. (FG).

Dado que en los textos periodísticos es necesario recabar información para desarrollar un tema, es que los integrantes de la comunidad de periodismo asocian la investigación de su área con la investigación científica o académica. A diferencia de esta última, en la indagación periodística no se describe el modo como se lleva a cabo la investigación.

El contexto en el que se realiza la tesis ocurre como parte de investigaciones mayores llevadas a cabo por los académicos de la carrera. De este modo uno de los estudiantes entrevistados señaló lo siguiente:

La investigación del seminario se enmarca en un proyecto más general de la escuela y del profesor guía que es la posibilidad de instalar un observatorio de medios acá en la escuela, entonces lo que estamos haciendo nosotros junto con el seminario para optar a nuestro grado académico. (E1 P).

En relación con la forma o estructura de la tesis, ella contiene las partes tradicionales de una investigación académica, a saber, planteamiento del problema, marco teórico, metodología, resultados, discusión y referencias bibliográficas. Así lo señalan los estudiantes:

[La tesis] partía con el nombre del proyecto, una descripción breve de no más de quinientas palabras, planteamiento del problema de investigación, planteamiento de la hipótesis, el planteamiento también de objetivos generales y específicos, después la especificación de toda la bibliografía recopilada. (E2 P).

En relación con la actividad de escribir, los integrantes de esta comunidad asocian dicha actividad como parte de su identidad tanto en la formación como en su desempeño profesional. Ello explica que, a diferencia de los hallazgos encontrados en las comunidades de ciencias del mar (TAPIA LADINO; MARINKOVICH, 2011), los estudiantes de esta comunidad no enfrentan la tarea de escribir como una dificultad o como expresión de una tensión. Al contrario, comprenden que escribir es una actividad que les otorga el sello a su profesión, así lo señala uno de los participantes: 
El discurso escrito [...] te ofrece la posibilidad de la organización, es una carrera que está centrada en la escritura y corresponde al pilar fundamental de un plan de estudio, de una malla curricular de cualquiera carrera de periodismo. (FG).

Las razones que explican el fenómeno central identificado, es decir, aquella afirmación que logra explicar todos los dichos de los entrevistados, se basan en la idea de que los textos periodísticos presentan rasgos comunes y diferentes con los textos de investigación científica. Básicamente las diferencias las encuentran en el tipo de público que lee los textos. Una de las intervenciones del FG explica este hecho:

Pero la diferencia es que principalmente la investigación o la estructura científica, por llamarla así, está orientada a un público mucho más especializado y específico, mientras que yo creo que la profundidad de un texto científico evidentemente tiene que ser muchísimo mayor que la de un mensaje masivo. (FG).

Cuando se revisan las condiciones que intervienen en el desarrollo del fenómeno, se advierte que entre las variables que favorecen la ocurrencia del fenómeno se relacionan con los procesos de enseñanza-aprendizaje propios de la carrera de periodismo. Es decir, el dominio progresivo de los géneros periodísticos, sumado a la relevancia asignada a la claridad como rasgo en la composición y al uso de las metodologías cualitativas de investigación son variables que potencian la idea de la investigación periodística como símil de la investigación académica. Así lo podemos refrendar en lo siguiente:

Lo básico es la textualización, o sea, independiente de que sea un discurso narrativo, hablemos de un discurso argumentativo, expositivo, etc. yo creo que la claridad es lo principal, que el alumno sepa textualizar la idea que quiere decir. (FG).

Cuando revisamos las condiciones que intervienen negativamente en el fenómeno identificado, visualizamos las dificultades que encuentran los estudiantes en el mantenimiento de la coherencia entre las partes de la tesis y, especialmente, el problema derivado de la construcción de la metodología. Uno de ellos señala lo siguiente:

El tema de redacción no tengo problemas no me complica, lo que me complica un poco [...] es plasmar la metodología en la forma escrita, en la forma en que yo voy a tener que poner en escrito el resultado de la investigación. (E2). 
El hecho de que los tesistas de periodismo hayan presentado dificultades en la construcción de la metodología puede derivar del hecho de que en los textos periodísticos normalmente no dan cuenta de los modos como acceden a la información. Este hábito podría estar a la base de esta dificultad para escribir la metodología de la tesis, ya que como señalan la tarea de escribir no exige para ellos grandes desafíos.

En relación con las estrategias de acción que los integrantes de esta comunidad implementan para enfrentar la escritura, en general, y la producción de la tesis en particular, se destaca las acciones provenientes del currículum de formación así como las iniciativas personales de los estudiantes. Así se evidencia en las intervenciones tanto de los docentes como de los tesistas.

El resto de los informes ahí sí que yo creo que hay una debilidad sobre todo en el tema de escritura de documentos científicos que es lo que estamos viendo ahora nosotros nos vamos a ver en la obligación de tener unas clases específicas de redacción de documentos científicos que nos va a dar una profesora, porque [...] se evidenció esa debilidad [...] (E1).

Como se aprecia en el extracto, los apoyos para el desarrollo de competencias escriturales, por tanto, se relacionan con factores externos a los estudiantes, como un diseño en la malla de formación orientada a ese fin así como sistemas de evaluación y monitoreo y con factores internos, la motivación y el trabajo personal.

Las consecuencias que hemos identificado en esta primera codificación axial nos indican que existe una tensión entre los textos académicos y profesionales. Dicha situación trae como consecuencia una dificultad para disociar los rasgos de una y otra y como resultado una suerte de traslapo entre los tipos de escritos, así lo señala el siguiente participante:

[...] en el fondo escribir para publicaciones, para artículos con una finalidad más que nada académica, porque a veces [...] existe una especie de confrontación en ese sentido y el alumno muchas veces termina o escribiendo como un reportaje, un artículo académico, por lo tanto no hay una claridad de estilo muchas veces. (FG).

Como se advierte las similitudes que encuentra la comunidad entre la tesis final de la carrera con las investigaciones periodísticas y los factores que inciden en la formación, traen como consecuencia la dificultad para distinguir entre el discurso académico y el discurso profesional. 
En cuanto a las representaciones sociales de la comunidad académica de la carrera de Trabajo Social acerca de la escritura de la tesis, una vez realizada la codificación axial de lo expresado por ellos, se esboza el siguiente esquema:

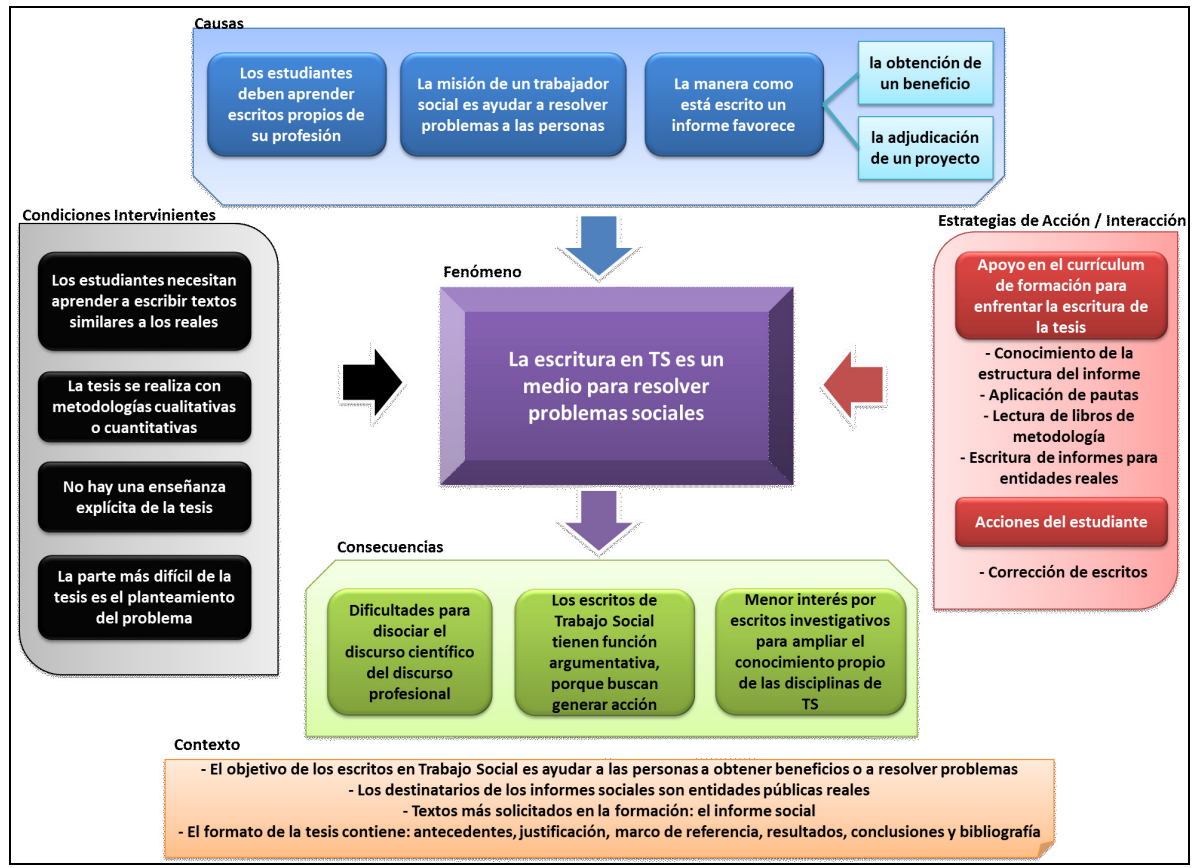

FIGURA 2 - La tesis en TS aborda la resolución de problemas sociales Fuente: Elaboración propia.

Como advertimos en el análisis, los integrantes de la carrera de Trabajo Social asumen la escritura como una manera de extender su función profesional, es decir, solucionar problemas sociales. Se advierte que la escritura forma parte de una actividad que es la extensión de la función propia de su desempeño profesional. Así lo evidencian los comentarios de los entrevistados, de los que seleccionamos la siguiente intervención:

Las tesis, el texto de la tesis es como de investigaciones sociales, sirve para después a lo mejor generar proyectos, sirve para, aplicar a lo mejor algún, algún proyecto como te decía e alguna población, en alguna comunidad y en seguida es de los informes o de los proyectos es como para validar el trabajo que tú estás haciendo. (E1). 
El desarrollo de la tesis tiene como precedentes los escritos de textos que tienen como fin ayudar a las personas a resolver algún problema o a obtener algún beneficio. Como el informe social es el tipo de texto que más debe desarrollar un trabajador social en su entorno profesional, el diseño de formación de la carrera favorece y promueve la elaboración de estos textos. Normalmente los destinatarios de estos escritos son entidades reales a quienes la carrera de Trabajo Social presta servicio con informes sociales.

En lo práctico, escribimos informe socioeconómicos, sociales, eso es pan nuestro de cada día en la carrera. Uno los genera por diversos motivos, ya sea para dar cuenta de la situación de una persona ante tribunales, para solicitar la adquisición de algún beneficio [...] estatal, privado, etc. (E2).

En cuanto a los rasgos de la tesis, ella presenta las partes canónicas: un marco teórico, metodología, resultados y conclusiones. Así lo señalan los académicos durante el FG: "tiene un desarrollo metodológico ligado a la recopilación, cotejamiento y tratamiento del análisis y obviamente una conclusión.”

Las causas que explican que la escritura esté orientada a la ayuda social se relacionan con la necesidad de proveer a los estudiantes una formación que simule situaciones del ejercicio de la profesión. Es decir, como en toda carrera universitaria una manera de demostrar el desempeño es mediante la elaboración de textos propios del mundo profesional. Las decisiones formativas de la carrera se relacionan con la idea del impacto real que tiene el desempeño del trabajador social sobre el destino de las personas, por lo tanto ello vuelve imperativo conocer y dominar los escritos propios de la profesión.

[...] de la buena expresión de ciertas ideas en un informe va a depender si una familia, permanece o no con un hijo, si se entienden los argumentos para evaluar una pensión de alimentos, si un proyecto se gana o no se gana. (FG).

Para enfrentar esta necesidad, la carrera de Trabajo Social enseña a escribir trabajos similares a los reales durante la formación. Cuando abordan las investigaciones lo hacen utilizando metodologías cualitativas o cuantitativas.

Entre las condiciones que intervienen negativamente en el fenómeno identificado, se advierte la dificultad que encuentran los tesistas para escribir el planteamiento del problema. 
[...] hay una resistencia muy marcada en el proceso de tesis a todo lo que es el planteamiento del problema, ubicarse en una perspectiva de ver las otras que existen, habitualmente yo creo que hay una resistencia. (FG).

Cuando revisamos las estrategias de acción para favorecer el desarrollo de escritos a la solución de problemas, se advierte, al igual que en la comunidad de periodismo, que los apoyos son externos e internos. Unos provienen de la estructura curricular de la carrera que se encarga de proveer apoyos como el conocimiento de los formatos, la presentación de pautas, la revisión de textos de metodología y la escritura de informes sociales para entidades reales. Las estrategias internas tienen que ver con la capacidad de los tesistas de corregir sus escritos a la luz de las observaciones de sus profesores guías.

El libro de cada uno, o sea como cada uno va armándose uno, o sea lo cuantitativo, lo cualitativo, cada uno tiene su orden. Entonces cada uno va viendo según lo que nos sirve, pero como que los profesores, por ejemplo mi profesor guía, nos da pautas, que tenemos que ir buscando las muestras, después ir buscando los objetivos, así como súper pauteado. (E2).

Hay autores que escriben sobre cómo realizar una investigación y te van dando como todos los pasos. (E2).

Como consecuencia de los factores antes descritos, podemos señalar que en el caso de Trabajo Social, la comunidad encuentra dificultades para disociar el discurso científico del discurso académico. Desde esta perspectiva, se advierte el fuerte influjo que recibe la carrera de su medio externo, es decir, la carrera hace suyo el deber de preparar a sus estudiantes en el desempeño académico lo más centrado en el mundo profesional. Esa determinación parece ir en desmedro de que la carrera desarrolle sus saberes disciplinares que sea necesario desarrollar.

Los datos revisados, permiten señalar que para la carrera de Trabajo Social el desarrollo profesional determina lo que se escribe durante la formación. Una vez realizada la codificación axial, procede la codificación selectiva, proceso que significa la elección de una categoría nuclear que relaciona todas las categorías anteriores en torno a una categoría central. Tanto la codificación axial como la selectiva van construyendo una teoría formal, teoría de tipo emergente que se deriva de una teoría sustantiva que se alimenta directamente de los datos. 


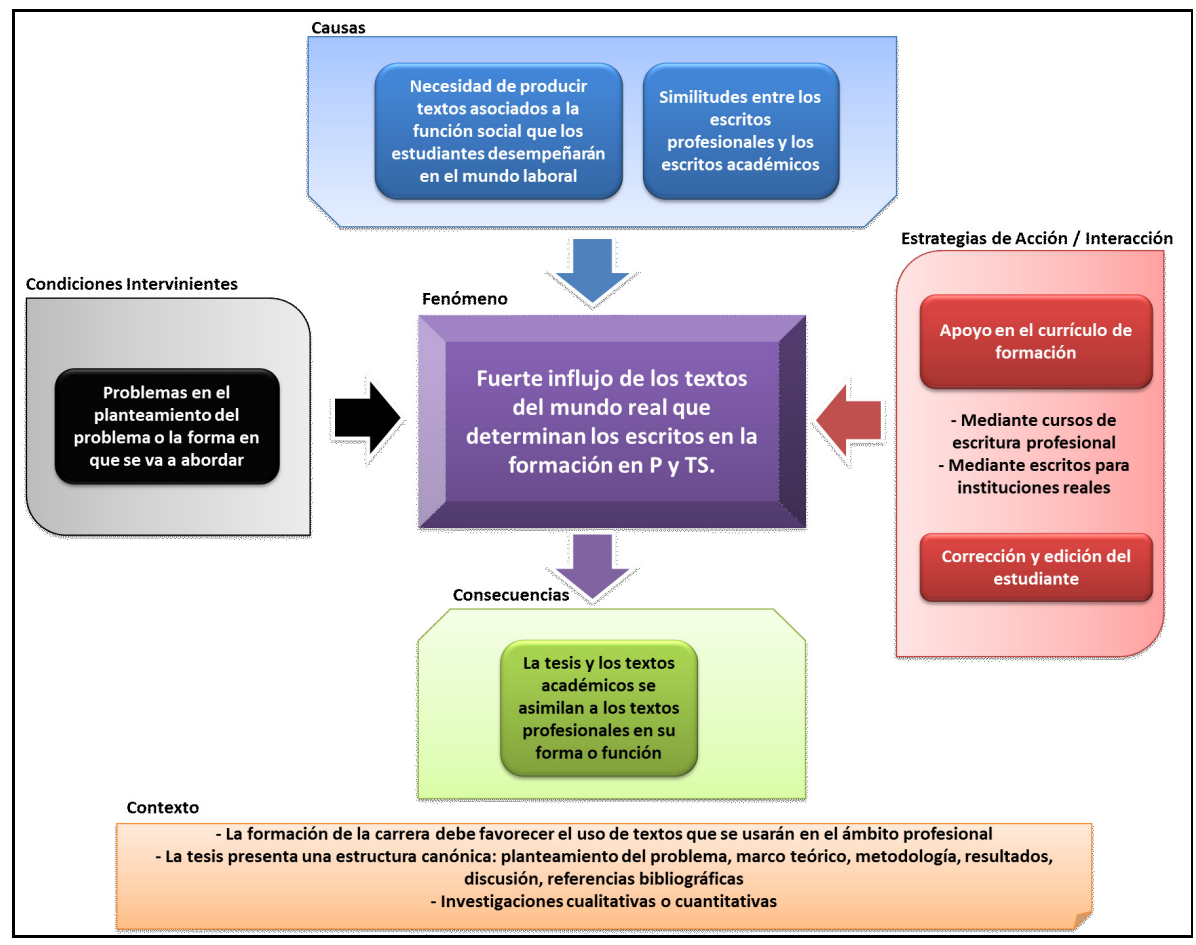

FIGURA 3 - Integración de las representaciones en torno a la escritura de la tesis en Periodismo y Trabajo Social

Fuente: Elaboración propia.

La categoría nuclear identificada a partir de las codificaciones axiales permite señalar que existe una determinación importante de los textos del mundo real hacia los textos de la formación de los estudiantes en las carreras analizadas. Parece ser que el influjo que producen los textos profesionales hacia los textos académicos determina la forma y función de los escritos que se escoge enseñar durante la formación. Aunque en ambas carreras la tesis presenta una estructura similar, cuestión que más adelante pretendemos refrendar con el análisis retórico-discursivo de las tesis de las carreras, la función asignada a ellas es diferente en cada carrera. En este sentido, a diferencia de los resultados obtenidos en una investigación anterior (TAPIA LADINO; MARINKOVICH, 2011) donde la tesis está altamente determinada por el género científico artículo de investigación, en el caso de las carreras estudiadas aquí son los textos profesionales los que definen el currículum de escritura en Periodismo y Trabajo Social. 
En la línea señalada anteriormente, estamos de acuerdo con Alcaraz Varó et al. quienes señalan que en la Universidad

se perciben dos movimientos epistemológicos complementarios, [...] el flujo de información hacia las profesiones y el reflujo proveniente de ellas. De esta forma la universidad da conocimientos e información lingüística a la sociedad, pero también recibe de ésta renovándose y perfeccionándose por el continuo contacto con la realidad que le ofrece este movimiento circular. (ALCARAZ VARÓ et al., 2007, p. 7).

Esta hipótesis plantea una relación de determinaciones mutuas entre los textos académicos y los textos profesionales, fenómeno que advertimos en esta investigación.

Para el caso estudiado, las causas que promueven esta determinación desde el mundo profesional parecen ser la similitudes encontradas en los tipos de investigación académica y profesional y, al mismo tiempo, la función que los textos juegan en el mundo real. La forma y la función que los integrantes de las comunidades asignan a los textos estarían a la base de la relación entre los escritos que se enseñan en la universidad y los textos que los futuros estudiantes deberán escribir en su desempeño profesional. La asociación entre investigación periodística e investigación científica y entre indagación social con el estudio de problemas reales revela la fuerte determinación del mundo real fuera de las fronteras universitarias. Las comunidades estudiadas encuentran espacios en el discurso académico para permear su propia identidad y, de ese modo, logran cumplir su rol.

En esta misma línea, estamos de acuerdo a Britt-Louise Gunnarsson (1997) cuando señala que los textos que se producen en las carreras están directamente asociados al rol que asumirán los estudiantes en el mundo profesional. La fuerte idea de que los textos generan prestigio profesional es una variable considerada por las comunidades para asegurar la calidad de su formación. Un buen profesional debe demostrar que es capaz de elaborar textos similares a aquellos que escribirá en el mundo laboral y ese desempeño se prueba en la universidad, no fuera de ella. Este hecho explica que las carreras enseñen a escribir explícitamente durante la formación los textos profesionales mediante la implementación de los cursos de escritura profesional o mediante la producción de escritos a lectores o instituciones reales. De este modo, la forma como se construye el currículo de las carreras estudiadas exige la generación de espacios comunicativos verosímiles como estrategias que favorecen la producción de 
textos académico-profesionales. Asimismo, el trabajo personal del estudiante y la fuerte identidad que expresa con su profesión explican la voluntad que colocan en mejorar la calidad de sus escritos. La corrección y edición constante son las acciones comunes entre los estudiantes de Periodismo y de Trabajo Social para enfrentar los escritos con buenos resultados.

Como consecuencia de lo señalado hasta ahora, en las comunidades estudiadas se advierte una asimilación de la forma y función de la tesis y los textos producidos durante la carrera con los textos profesionales. La forma y función de dichos textos evidencia el espacio intermedio que ocupan entre el mundo académico y el mundo laboral. La tesis de Periodismo es concebida como una investigación científica y la tesis de Trabajo Social tiene la aspiración de favorecer la solución de un problema social. En ambos casos el desempeño profesional futuro de los estudiantes determina qué y cómo se escribirá la tesis. Los textos precedentes en la formación también juegan un rol preparatorio en este sentido. El análisis de las representaciones sociales en las carreras estudiadas nos permite señalar que la escritura de los textos académico-profesionales es asumida como parte de un continuo donde se perciben los textos académicos como artefactos de prestigio social.

\section{A modo de conclusión}

Para las comunidades estudiadas, la producción de la tesis y los escritos propios de la formación están fuertemente determinados por el medio profesional. Se trata de una visión de la escritura profesional como un influjo externo que determina las acciones que suceden al interior de la formación universitaria. Se advierte entonces la idea de que el dominio de los textos del ámbito laboral puede asegurar la calidad del desempeño de los futuros profesionales. Por lo tanto, advertimos que los escritos profesionales gozan de un prestigio tal que son lo suficientemente valorados como para ser asumidos en la formación. Llama la atención en ambas carreras la escasa presencia de la idea del desarrollo de conocimientos disciplinares. Es posible que esta ausencia se relacione con formaciones universitarias aplicadas a la realidad donde las disciplinas de formación parecieran estar al servicio de la sociedad. De esta manera hemos constado que existe una representación de la escritura orientada a la acción en el mundo real.

Nuestras conclusiones son diferentes a las halladas en una investigación anterior donde advertimos en el área de ciencias ausencia de textos profesionales y una preponderancia de textos científicos, específicamente, el artículo 
científico (TAPIA LADINO; MARINKOVICH, 2011). Para las carreras estudiadas en esta oportunidad advertimos un movimiento inverso, una valoración de los textos profesionales por sobre aquellos que den cuenta de hallazgos científicos orientados al desarrollo disciplinar. El fuerte influjo que reciben las carreras de Periodismo y Trabajo Social del mundo profesional, se hace evidente en el modo como dichas comunidades conciben el trabajo final de titulación. La correspondencia entre tesis e investigación periodística y entre tesis y estudio socioeconómico revela el alto compromiso que los programas de formación tienen con su entorno laboral. Nos queda ahora acceder a la descripción de las tesis y artículos que producen las comunidades estudiadas con el fin de averiguar y, en definitiva, triangular nuestros hallazgos con el análisis de los textos.

\section{Referencias}

ADELSTEIN, A. Representación de unidades terminológicas: el modelo de término wüsteriano. Organon, Porto Alegre, n. 26, p. 67-89, 1998.

ALCARAZ VARÓ, E.; MATEO, J.; YUS, F. (Ed.). Las lenguas profesionales y académicas. Barcelona: Ariel Lengua Moderna, 2007.

ALVAREZ, C. A. Los jóvenes, las drogas y el alcohol: un estudio de sus representaciones sociales. Documentos CIDE, Santiago (CL), v. 96, n. 1, 1996. ARNOUX, E. N. de. Escritura y producción de conocimiento en las carreras de posgrado. Buenos Aires: Santiago Arcos, 2009.

ARNOUX, E. N. de; ALVARADO, E.; BALMAYOR, E.; DI STEFANO, M.; PEREIRA, C.; SILVESTRI, A. Talleres de lectura y escritura. Buenos Aires: Eudeba, 1998. ARNOUX, E. N. de; DI STEFANO, M.; PEREIRA, C. La lectura y la escritura en la universidad. Buenos Aires: Eudeba, 2002.

BAZERMAN, C.; LITTLE, J.; BETHEL, L.; CHAVKIN, T.; FOUQUETTE, D.; GARUFIS, J. Reference Guide to Writing Across the Curriculum. Indiana: Parlor, 2005.

BONO, A.; DE LA BARRERA, S. La producción de escritos académicos de los estudiantes universitarios: análisis de aspectos conceptuales y estratégicos. Revista Con-textos de Educación, v. 1, n. 1, p. 130-137, 1998.

CANO, N. B. ¿Cómo generar la función epistémica del texto escrito en el aula escolar? Lectura y Vida: Revista Latinoamericana de Lectura, v. 20, n. 1, p. 14-21, 1999.

CARLINO, P. Escribir, leer y aprender en la universidad: una introducción a la alfabetización académica. Buenos Aires: Fondo de Cultura Económica, 2005. 
CASSANY, D. Investigaciones y propuestas sobre literacidad actual: multiliteracidad, Internet y criticidad. In: CONGRESO NACIONAL CÁTEDRA UNESCO PARA LA LECTURA Y LA ESCRITURA, 1., 24-26 ago. 2005, Concepción. Actas... Concepción: Universidad de Concepción, 2005. Disponible en: <http:// www2.udec.cl/catedraunesco/05CASSANY.pdf>. Acceso en: 22 dec. 2012.

CASSANY, D. Para ser letrados: voces y miradas sobre la lectura. Barcelona: Paidós, 2009.

CAVAlLO, G.; CHARTIER, R. Historia de la lectura en el mundo occidental. Barcelona: Taurus, 1998.

DEL ROSAL, G. V. Los letrismos en la configuración de las comunidades de aprendizaje de posgrado. In: ARNOUX, E. N. de (Org.). Escritura y producción de conocimiento en las carreras de posgrado. Buenos Aires: Santiago Arcos, 2009. DURKHEIM, E. Représentations individuelles et représentations collectives. Revue de Métaphysique et de Morales, tome VI, p. 273-300, mai 1898.

ESPEJO REPETTO, C. La movida concluyendo en torno al tema en informes de investigación elaborados por estudiantes universitarios. Revista Onomázein, Santiago, v. 13, n. 1, p. 35-54, 2006.

GLASER, B.; STRAUSS, A. The Discovery of Grounded Theory. Chicago: Aldine, 1967. GONZÁLEZ, F.; RIVERA, M.; GALINDO, M.; GRANDÓN, O. Representaciones sociales sobre comprensión y producción de textos en alumnos de la Carrera de Pedagogía en Lenguaje y Comunicación de la Universidad de Antofagasta en Chile y de la Carrera de Licenciatura en Lingüistica y Literatura de la Universidad de Sonora en México. Hermosillo: Editorial Universidad de Sonora; Unesco; Universidad de Antofagasta, 2009.

GUNNARSSON, B. Applied Discourse Analysis. In: DIJK, T. van (Ed.). Discourse as Social Interaction. London: Sage, 1997. p. 285-314.

HARVEY, A. M. La evaluación en el discurso de informes escritos por estudiantes universitarios chilenos. In: PILLEUX, M. (Ed.). Contextos del discurso. Valdivia: Universidad Austral de Chile, 2005. p. 215-228.

HARVEY, A. M.; MUÑOZ, D. El género informe y sus representaciones en el discurso de los académicos. Revista de Estudios Filológicos, Valdivia, n. 41, p. 95-114, sep. 2006.

JODELET, D. D. Représentations sociales: un domaine en expansion. In: . (Ed.). Les représentations sociales. Paris: Presses Universitaires de France, 1989. p. 31-62.

LEMKE, J. L. Articulating Communities: Sociocultural Perspectives on Science Education. Journal of Research in Science Teaching, v. 38, n. 3, p. 296-316, mar. 2001. 
MARINKOVICH, J.; MORÁN, P. La escritura a través del currículum. Revista Signos, Valparaíso, v. 31, n. 43-44, p. 165-171, 1998.

MARINKOVICH, J.; VELÁSQUEZ, M. La representación social de la escritura y los géneros discursivos en un programa de Licenciatura: una aproximación a la alfabetización académica. In: Parodi, P. (Ed.). Alfabetización académica y profesional en el siglo XXI: leer y escribir desde las disciplinas. Santiago (CL): Academia Chilena de la Lengua; Planeta, 2010. p. 127-152.

McCORMICK, L. Didáctica de la escritura. Buenos Aires: Aique, 1997.

MOSCOVICI, S. On Social Representation. In: FORGAS, J. P. (Ed.). Social Cognition: Perspectives in Everyday Life. London: Academic Press, 1981. p. 181-210.

MOSCOVICI, S. Social Influence and Social Change. London: Academic Press, 1976.

MURRAY, D. M. The Craft of Revision. Fort Worth: Harcourt Brace College Publishers, 1997.

NÚNEZZ, P. Hacia una caracterización discursiva de los informes escritos por universitarios. In: SEGUNDO CONGRESO INTERNACIONAL Y QUINTO NACIONAL SOBRE LENGUA ESCRITA Y TEXTOS ACADÉMICOS, 2004, Universidad Autónoma de Tlaxcala, Tlaxcala.

NÚNEEZ, P.; ESPEJO, C. Estudio exploratorio acerca de la conceptualización del informe escrito en el ámbito académico. In: HARVEY, A. M. (Ed.). En torno al discurso: contribuciones de América Latina. Santiago (CL): Ediciones Pontificia Universidad Católica, 2005. p. 135-148.

PADRÓN, J. Análisis del discurso e investigación social. Caracas: Publicaciones del Decanato de Postgrado, Universidad Católica Santa Rosa, 1996.

PARODI, G. (Ed.). Alfabetización académica y profesional en el siglo XXI: leer y escribir desde las disciplinas. Santiago (CL): Academia Chilena de la Lengua; Planeta, 2010.

PARODI, G. Géneros académicos y géneros profesionales. Accesos discursivos para saber y hacer. Valparaíso: Ediciones Universitarias de Valparaíso, 2008.

ROMERO, F. ¿Qué leen los estudiantes de la Universidad Tecnológica de Pereira? Ciencias Humanas, v. 5, n. 16, p. 120-130, jun. 1998.

ROMERO, F. La escritura en los universitarios. Ciencias Humanas, n. 21, 2000. Disponible en: <http://www.utp.edu.co/ - chumanas/revistas/revistas/rev21/ romero.htm>. Acceso en: 22 dec. 2012.

RUIZ, J. I. Metodología de la investigación cualitativa. Bilbao: Universidad de Deusto, 1996. 
STRAUSS, A.; CORBIN, J. Bases de la investigación cualitativa. Técnicas y procedimientos para desarrollar la teoría fundamentada. Bogotá: CONTUS, 2002.

SWALES, J. Genre Analysis: English in Academic and Research Settings. Cambridge (UK): Cambridge University Press, 1990.

TAPIA LADINO, M.; BURDILES, G.; ARANCIBIA, B. Aplicación de una pauta diseñada para evaluar informes académicos universitarios. Revista Signos, Valparaíso, v. 36, n. 54, p. 249-257, 2003.

TAPIA LADINO, M.; MARINKOVICH, S. Representaciones sociales sobre la escritura de la tesis en la formación académica inicial en el área de las Ciencias del Mar. Revista Onomázein, v. 24, n. 2, p. 273-297, 2011. Disponible en: <http://www.onomazein.net/24/12.pdf>. Acceso en: 22 dec. 2012.

TAPIA-LADINO, M.; SILVA, V. Mejorando la calidad de los escritos: una experiencia pedagógica universitaria en ámbito de la escritura en las disciplinas. Folios, n. 31, p. 91-104, primer semestre 2010.

TOLCHINSKY, L. Distintas perspectivas acerca del objeto y propósito del trabajo y reflexión metalingüística en la escritura académica. In: MILIAN, M.; CAMPS, A. (Ed.). El papel de la actividad metalingüistica en el aprendizaje de la escritura. Rosario: Homo Sapiens, 2000.

TRINIDAD, A., CARRERO, V.; SORIANO, R. Teoría fundamentadaGrounded Theory: la construcción de la teoría a través del análisis interpretacional. Madrid: CIS, 2006.

VENEGAS, R. Clasificación de textos académicos en función de su contenido léxico-semántico. Revista Signos, Valparaíso, v. 40, n. 63, p. 239-271, 2007.

ZAVALA, V. La literacidad o lo que la gente hace con la lectura y la escritura. In: CASSANY, D. Para ser letrados: voces y miradas sobre la lectura. Barcelona: Paidós, 2009. p. 23-36. 